\title{
Proceedings of the 2007 meeting of the Scottish Otolaryngological Society, Dunkeld Hilton, Dunkeld, Scotland, UK, 10 May 2007
}

\section{Comparison of external and endonasal dacryocystorhinostomy}

M Feretis, J R Newton, F D Green, B Ram

From the Aberdeen Royal Infirmary, Scotland, UK

\section{Introduction}

External dacryocystorhinostomy (DCR) has been the treatment for nasolacrimal duct obstruction for more than a century. More recently, nasal endoscopy has allowed this procedure to be carried out endonasally.

Aim

The aim of this postal questionnaire study was to compare the health status of patients treated for epiphora by external versus endonasal non-laser DCR.

\section{Method}

All patients fitting our criteria were sent a copy of the Glasgow Benefit Inventory questionnaire, along with an additional, validated questionnaire regarding their symptoms.

Results

Questionnaires were satisfactorily completed by 64 patients out of 90 in the external DCR group and by 30 patients out of 41 in the the endonasal DCR group. The results indicated positive scores for both groups for all four subscales of the Glasgow Benefit Inventory. There were no significant statistical differences between the external and endonasal procedures. Ocular symptomatology scores were better for the external DCR patients but, again, this difference did not reach statistical significance.

\section{Appropriate antibiotics for peritonsillar abscess: a nine-month cohort}

N Kara, C Spinou

From the Ninewells Hospital \& Medical School, Dundee, Scotland, UK

\section{Objectives}

To assess the efficacy of our current departmental protocol for the management of peritonsillar abscess, within a tertiary ENT referral centre.

Methods

Seventy-eight patients were referred to the department with a peritonsillar abscess over a nine-month period. Patients' clinical courses were charted and their aspirates sent for culture and sensitivity testing.

Results

Fifty-two cases of peritonsillar abscess were confirmed. Twenty patients received monotherapy, while 30 patients were also given metronidazole. The use of metronidazole was appropriate in two-thirds of cases and unnecessary in one-third. One-third of patients who required metronidazole were not prescribed it, resulting in a longer in-patient stay of 2.3 days compared with 1.9 days.

\section{Conclusions}

The frequent finding of mixed anaerobes as sole or second organisms justified the use of metronidazole in our department. We recommend a combination of intravenous penicillin and metronidazole for initial treatment of all patients with a peritonsillar abscess.

\section{Impact of intensive care admission on vocal function}

I Nixon, S Ramsay*, K MacKenzie

From the Departments of Otolaryngology, Head and Neck Surgery and *Anaesthesia, North Glasgow Division, Scotland, UK

\section{Objective}

To characterise the extent of perceived vocal morbidity following discharge from intensive care.

\section{Method}

Prospective study including 273 patients discharged from an intensive care unit. All live patients were sent a copy of the Voice Symptom Scale questionnaire. This process was repeated after one month for all patients who had not responded.

\section{Results}

Results were as follows: 273 patients were identified; 187 patients were included; 86 patients responded; three patients refused to consent to be involved; 83 (46 per cent) patients consented to be involved. For these patients, the median age was 62 years, the median total Voice Symptom Scale score was 12 , and the interquartile range was six to 25.25. Thirty-three per cent of respondents scored $>20$, while 16 per cent scored $>40$.

\section{Conclusions}

Thirty-three per cent of patients discharged from an intensive care unit had a Voice Symptom Scale score similar to or worse than that of patients treated for early laryngeal cancer. Ten per cent of patients had a Voice Symptom Scale score similar to or worse than that of patients attending the voice clinic.

\section{Audit of endoscopic dacryocystorhinostomy}

G Kulandaivelu, E Stewart

From the Monklands Hospital, Airdrie, Scotland, UK

Aim

To review the results of endoscopic dacryocystorhinostomy (DCR) for the Lanarkshire National Health Service trust.

\section{Patients and methods}

We conducted a retrospective review of 78 endoscopic DCRs (65 primary and 13 revision) performed between April 2004 and December 2005. Silicon stents were used in all cases. The patients were followed up at six months when the stents were removed. 
Results

Fifty-nine of the 74 operations for which results were documented resulted in patients experiencing either complete relief or an improvement following the procedure, giving an overall success rate of 79.7 per cent. Complications were minor. The failure rate was found to be higher in those who had experienced problems with stenting.

\section{Discussion}

The success rate in our unit was comparable to the standard set in guidelines published by the National Institute of Clinical Excellence. Our experience with stents suggests that, contrary to some reports, they may have contributed, at least partly, to the success of this operation.

\section{Identifying snorers with obstructive sleep apnoea: how hard can it be?}

S Robertson, D Young*, G W McGarry, K MacKenzie

From the Glasgow Royal Infirmary and the *University of Strathclyde, Glasgow, Scotland, UK

\section{Aims}

To determine the value of the Epworth score, body mass index (BMI), and history of witnessed apnoeas or choking episodes, as predictors of obstructive sleep apnoea/hypopnoea syndrome in disruptive snorers.

\section{Methods}

A structured history was obtained by clinic interview from 100 patients referred with disruptive snoring. All patients underwent detailed ENT examination and were triaged according to guideline 73 of the Scottish Intercollegiate Guidelines Network. ${ }^{1}$ Univariate and receiver operating characteristic analyses were undertaken on data collected.

\section{Results}

There was a significant association between the Epworth score and a diagnosis of obstructive sleep apnoea/hypopnoea syndrome $(p=0.033)$. However, the cut-off recommended by the Scottish Intercollegiate Guidelines Network for Epworth score $(>10)^{1}$ yielded a diagnostic test with poor sensitivity and specificity. There was no association between the presence of witnessed apnoeas or choking episodes, or a BMI of $>30$, and a diagnosis of obstructive sleep apnoea/hypopnoea syndrome.

\section{Conclusion}

This study does not support the use of a specific Epworth score cut-off as a trigger for sleep study investigation. Elevated BMI and a history of witnessed apnoeas or choking episodes are similarly non-specific for obstructive sleep apnoea/ hypopnoea syndrome. The diagnostic role of the ENT surgeon in first-line snoring assessment remains limited.

\section{Reference}

1 Scottish Intercollegiate Guidelines Network. Management of Obstructive Sleep Apnoea/Hypopnoea Syndrome in Adults (Guideline 73). Edinburgh: Scottish Intercollegiate Guidelines Network, 2003.

\section{The challenge of the congenital nasal dermoid cyst}

\section{R Srivastava, M-L Montague*}

From St John's Hospital, Livingston, and the *Royal Hospital for Sick Children, Edinburgh, Scotland, UK

\section{Introduction}

Congenital midline nasal lesions are rare, occurring in one in 20000 to 40000 births. Of these midline lesions, nasal dermoids are the most common. Histologically, they comprise ectodermal and mesodermal components. Intracranial extension has been reported in up to 45 per cent of cases.

\section{Methods}

We present the case of a 13-month-old boy referred to our department with an infected, midline nasal swelling. We outline the initial management, differential diagnosis, and the role of imaging in diagnosis and surgical planning. We also detail the surgical approach employed in this challenging case.

Results

An extensive lesion with intracranial extension was delineated with high resolution computed tomography and multiplanar magnetic resonance scanning. Antibiotic therapy was commenced and continued as prophylaxis until surgery. Complete excision of the lesion was achieved via an external rhinoplasty approach, with repair of the skull base defect and resulting cerebrospinal fluid leak. The post-operative course was uncomplicated, and cosmesis was acceptable at three months' follow up.

\section{Conclusions}

Although nasal dermoids are rare, their potential for local bony atrophy and distortion of the nose, as well as for serious infections such as meningitis and brain abscess, warrants early recognition, appropriate imaging to permit accurate diagnosis, and prompt treatment.

\section{Patients' satisfaction with a nurse-led aural care clinic}

M Shakeel, J Newton, D Clark, A Hussain

From the Aberdeen Royal Infirmary, Scotland, UK

\section{Introduction}

Nurse-led clinics are becoming increasingly common in otolaryngology. This clinic was run by an experienced nurse, with medical support available on demand.

\section{Aim}

To assess patients' satisfaction with this nurse-led aural care clinic.

\section{Materials and method}

Patients attending the clinic were requested to fill in an eight-item, anonymous questionnaire. Data were collected over a 14-week period from January to April 2007. The Statistical Package for the Social Sciences version 12 software was used for analysis.

\section{Results}

Forty-four questionnaires were completed fully $(44 / 50=$ 88 per cent). In response to patients' queries or concerns, the nurse gave verbal advice $(n=25)$, discussed the case with a doctor $(n=14)$ or arranged a doctor review $(n=$ 17). Forty patients were very satisfied and four were satisfied with the overall care in the clinic.

\section{Discussion}

Combining medical and nursing efforts can enable efficient use of limited time resources within the National Health Service. Nurse-led clinics are cost-effective and help reduce the waiting time for out-patient clinic appointments. 

Submandibular gland excision: does the primary surgeon's
training level affect the complication rate?

K McAllister, M Supriya, F Ahsan, L McClymont

From the Raigmore Hospital, Inverness, Scotland, UK

Objective

To review the results of submandibular gland excision, assessing the primary surgeon's level of training and the effect of this on complication rates.

\section{Methods}

Retrospective analysis assessing the results of submandibular gland excision over an 11-year period (May 1994 to October 2005) at the Raigmore Hospital.

\section{Results}

Approximately two-thirds (57 per cent) of all patients were operated upon primarily by registrars, the remainder by consultants. The overall complication rates were 22 per cent for registrars versus 14 per cent for consultants. The rate of facial nerve palsy was higher for registrars than for consultants (11 vs 4 per cent, respectively; all temporary) as was the infection rate ( $3 v s$ per cent, respectively). However, the haematoma rate was comparable (8vs 7 per cent, respectively). The registrars had a lower rate of sialocoele ( 0 vs 4 per cent, respectively).

\section{Discussion}

There was no significant difference between the complication rates of registrars and consultants. Our complication rates were comparable to accepted standards. Our rate of temporary marginal mandibular branch paralysis was 8 per cent, as compared with 11-13 per cent for Bradley (2001), 12 per cent for O'Brien et al. (1998) and 36 per cent for Smith et al. (1993).

\section{Self-assessment of nasal injury by patients: is it reliable?}

D E C Baring, R Locke, S Kumar*

From St John's Hospital, Livingston, Scotland, and *private practice, Milton Keynes, England, UK

\section{Introduction}

In our weekly fractured nose clinic, it was noted that there was a high non-attendance rate and that many referrals did not need treatment. A study was designed to assess whether patients could safely self-assess their injury and self-refer if required.

\section{Methods}

Forty-nine consecutive nasal injuries were seen in the fractured nose clinic at St John's Hospital, Livingston, Glasgow, from April to July 2005. The patients were asked to self-assess the extent of their swelling, deformity, obstructed airway or persistent discharge. Their answers were sealed prior to assessment. The results were then compared to those of the clinic assessment.
Results

In the initial period, 61 patients were referred and 49 attended (i.e. 20 per cent non-attendance rate). Twentythree (47 per cent) patients were offered manipulation. The self-assessment comparison showed no effective correlation for each of the above parameters. However, 82 per cent $(19 / 23)$ of those offered manipulation would have arranged an appointment.

On this basis, an information leaflet was introduced which was given to patients on attendance at the accident and emergency department. One week after their injury, they were advised to self-assess and to telephone for an appointment if required.

\section{Discussion}

Patients can be relied upon to self-assess their nasal injury and to self-refer. The four patients who stated that they would not have done so had minor deformities and were only listed for manipulation after much discussion. Introduction of the patient information leaflet resulted in a reduced need for out-patient clinic appointments for nasal trauma, and also increased the appropriateness of attendance. Audit of this process is ongoing.

\section{Can we improve dysphagia referrals?}

S A Phillips, P D Ross, K Chalmers, G MacDougall

From the Edinburgh Royal Infirmary, Scotland, UK

Aim

We set out to examine whether a multidisciplinary outpatient dysphagia referral triage service would shorten the duration of a patient's referral process and direct patients to the correct specialty.

Methods

A review was carried out of patients referred with dysphagia before and after the introduction of a multidisciplinary out-patient dysphagia service, from February 2001 to April 2001 and from January 2002 to March 2002, inclusive. A total of 108 patients was referred.

\section{Results}

The length of time until the first appointment was reduced from four to three weeks (median; range one to 23 weeks; $p=0.001$ ). The number of instrumental investigations was reduced, with a median of one instrumentation per patient under the new service, compared with two per patient under the old service $(p=0.001)$. Attendance to hospital was also reduced, with 45 per cent of patients under the new service requiring only one appointment, compared with 13 per cent of those under the old service $(p=0.001)$.

\section{Conclusions}

Introduction of a multidisciplinary out-patient dysphagia service was associated with significant reductions in waiting times, in the number of instrumental investigations and in the duration of the patient's referral process. 